\title{
Predictors of response to infliximab in patients with fistulizing Crohn's disease
}

\author{
M. Luna-Chadid, J.L. Pérez Calle', J.L. Mendoza², M. I. Vera³ , A. F. Bermejo ${ }^{4}$, F. Sánchez ${ }^{5}$, A. López San \\ Román $^{4}$, C. Froilán ${ }^{6}$, V. González-Lara ${ }^{1}$, J. García-Paredes², I. Fernández-Blanco ${ }^{6}$, L. Abreu ${ }^{3}$, B. Casis ${ }^{5}$, \\ J. A. Solís Herruzo ${ }^{5}$ J.P. Gisbert and J. Maté-Jiménez
}

Hospital de La Princesa. ${ }^{1}$ Hospital Gregorio Marañón. ${ }^{2}$ Hospital Clínico. ${ }^{3}$ Hospital Puerta de Hierro. ${ }^{4}$ Hospital Ramón y Cajal. ${ }^{5}$ Hospital Doce de Octubre. ${ }^{6}$ Hospital La Paz. Madrid, Spain.

\begin{abstract}
Objective: to evaluate the efficacy and toxicity of infliximab for the treatment of fistulizing Crohn's disease.

Methods: consecutive patients with fistulizing Crohn's disease receiving infliximab were prospectively enrolled. Partial response was defined as a reduction of $50 \%$ or more from base-line in the number of draining fistulae. Complete response was defined as the closure of all fistulae. The influence of different variables on the efficacy of infliximab was evaluated.

Results: 108 patients were included. The disease was inflammatory plus fistulizing in $18 \%$ and only fistulizing in $82 \%$. After the third infusion of infliximab the response was partial in $26 \%$ and complete in $57 \%$. Response (\%) rates (partial/complete) depending on fistula location were: enterocutaneous (25/68\%), perianal (35/60\%), rectovaginal (36/64\%), and enterovesical (20/40\%). None of the studied variables (including concomitant immunosuppressive therapy) correlated with efficacy of infliximab in the multivariate analysis. Incidence of adverse effects (21\%) depending on the dose of infliximab was: first dose (5.6\%), second (7.4\%), and third (11.1\%).

Conclusions: infliximab is an efficacious treatment for fistulizing Crohn's disease. Partial response was achieved in approximately one third of the patients, and complete response in more than half. No studied variable was predictive of response. Adverse effects were relatively infrequent and mild.
\end{abstract}

Key words: Crohn's disease. Inflammatory bowel disease. Infliximab. Fistulizing Crohn's disease. Fistula. Treatment.

Luna-Chadid M, Pérez Calle JL, Mendoza JL, Vera MI, Bermejo AF, Sánchez F, López-San Román A, Froilán C, GonzálezLara V, García-Paredes J, Fernández-Blanco I, Abreu L, Casis B, Solís Herruzo JA, Gisbert JP, Maté-Jiménez J. Predictors of response to infliximab in patients with fistulizing Crohn's disease. Rev Esp Enferm Dig 2003; 96: 379-384.

Recibido: 17-12-03.

Aceptado: 23-12-03.

Financial Support: This work was supported in part by grants C03102 (to RMO) and C03101 (to FSM) from the Instituto de Salud Carlos III.

Correspondencia: J. Maté. Servicio de Enfermedades Digestivas. Hospital Universitario de La Princesa. Diego de León, 62. 28006 Madrid.

\section{INTRODUCTION}

Infliximab was approved by the Food and Drug Administration in 1998 as a 3-dose regimen for the treatment of fistulizing Crohn's Disease (CD) (1). Treatment requires hospitalization, is expensive and is associated with severe side effects, including infusion reactions and infectious complications $(2,3)$. In addition, the mean duration of response to medication is 2-3 months, (3-5) often requiring retreatment at regular intervals.

The clinical and demographic parameters that determine the response or lack of response remain unknown. Some studies suggest a positive correlation between the concurrent use of immunosuppressive agents and response to infliximab in patients with inflammatory CD (6-8). However, the concurrent administration of an immunomodulator showed no short-term advantage in fistulizing $\mathrm{CD}(2,3,7,8)$.

Identifying predictors of response to infliximab would be of great benefit in the selection of patients for this treatment, and this could in turn affect cost-related issues. The aim of this study was to evaluate the efficacy and to identify predictors of response to infliximab in fistulizing CD.

\section{METHODS}

Study subjects. All the consecutive CD patients who were treated with intravenous infusions of infliximab $5 \mathrm{mg} / \mathrm{kg}$ at seven University Hospitals in Madrid, from October 1999 to March 2001, were evaluated in a prospective cohort study.

Inclusion criteria. a) age $>18$ and $<65$ years; b) $\mathrm{CD}$ patients with one or more open fistulas; c) patients receiving medications for fistulizing $\mathrm{CD}$ (azathioprine/6-mercaptopurine, metronidazole, ciprofloxacin) for 6 months or longer; d) three infliximab infusions completed at 0,2 , and 6 weeks; and e) at least 4 weeks of post-infusion follow-up from the third infliximab infusion. 
Exclusion criteria. a) previous treatment with infliximab; b) serious infection, including tuberculosis; c) positive pregnancy test; and d) serious allergy history.

Study approval was granted by the respective Ethics Committees of the participating institutions. All patients gave written informed consent before study entry.

Data collection. The following data were collected: sex, age, concurrent medications, disease duration, smoking history, and intestinal disease location. Response was assessed by a face-to-face interview which was held 4 weeks after the third infusion.

Fistulas were classified into the following groups: enterocutaneous, perianal and internal, rectovaginal, and enterovesical fistulas. Patients receiving azathioprine/6mercaptopurine were started on immunosuppressive therapy more than 6 months before the first infliximab infusion. Smokers were defined as subjects smoking a minimum of 5 cigarettes per day. Non-smokers were defined as subjects who had never smoked or subjects who had quit smoking at least 6 months before the first infusion.

Classification of response. Complete response was defined as the complete cessation of drainage from all fistulas despite gentle finger compression. Partial response was defined as at least $50 \%$ reduction from baseline in the number of fistulas or drainage for at least 4 consecutive weeks after the discontinuation of drug infusions. For patients with rectovaginal fistulas, response was defined as closure documented by physical examination.

Statistical analysis. For quantitative variables, mean and standard deviation were calculated. For qualitative variables, percentage and $95 \%$ confidence interval were provided. Comparisons between independent proportions Were carried out by Chi square test. Quantitative variables were compared using Student's t-test. A multiple logistic regression analysis was performed to study the association between the efficacy of infliximab (expressed in two different ways: complete response $v s$ partial response or no response, and complete or partial response vs no response) and a number of variables: age (years), gender (male/female), smoking (smokers/non-smokers), duration of fistulizing disease (years), location of fistulas (enterocutaneous, perianal, and internal fistulas), spontaneous/postoperative nature, and concomitant immunosuppressive therapy. We used a backward modelling strategy. Log-likelihood ratio was the statistic used for model comparison.

\section{RESULTS}

Demographics. One hundred and eight patients were enrolled (mean age $38 \mathrm{yr}, 53 \%$ men, 50\% smokers). The mean duration of disease was $9 \mathrm{yr}$, and the mean duration of fistulizing disease was $5.4 \mathrm{yr}$. The disease was inflammatory plus fistulizing in $18 \%$, and only fistulizing in $82 \%$ of the cases. The disease location was ileal in $31 \%$, colonic in $18 \%$ and ileocolonic in $51 \%$ of the cases. The percentages of patients receiving concurrent therapy with other drugs were: azathioprine/6-mercaptopurine (68\%), corticosteroids $(55 \%)$, 5-aminosalicylates $(75 \%)$, metronidazole $(67 \%)$, and ciprofloxacin $(32 \%)$. The number of fistulas in each patient ranged from 1 to 6 (mean 1.8). The number of fistulas by location was: 24 enterocutaneous, 59 perianal, and 12 internal (rectovaginal or enterovesical) fistulas.

Response. One hundred and five patients were finally evaluated for the study, 3 patients $(2.7 \%)$ being excluded for not undergoing post-treatment check-up. After the third infusion of infliximab, 87 patients $(82 \%)$ were responders: the response was partial in $26 \%$ and complete in $57 \%$ of the cases. Response rates (partial/complete, \%) by location of fistulas were: enterocutaneous (25-68\%), perianal $(35-60 \%)$, rectovaginal $(36-64 \%)$ and enterovesical $(20-40 \%)$.

The characteristics of studied variables in CD patients with and without response to infliximab are shown in table I. After adjusting for age at infusion, disease duration, and sex, the logistic regression analysis showed that the variables studied (smoking, duration of fistulizing disease, fistula location, spontaneous/surgical nature, and concomitant immunosuppressive therapy) did not affect the rate of response.

Adverse effects were reported in 22 patients (21\%): flulike symptoms (3 patients), pruritus (2), headache (1), nausea (4), vomiting (2), hypotension (3), pneumonia (2), fever (1), thrombocytopenia (1), hyperbilirubinemia (1), anaphylactic reaction (2), and tuberculosis (1). Adverse effects (\%) depending on the dose of infliximab infusion were: first, $5.6 \%$, second, $7.4 \%$, and third infusion, $11.1 \%$.

\begin{tabular}{|c|c|c|c|}
\hline Variable & Responders & $\begin{array}{l}\text { Non- } \\
\text { responders }\end{array}$ & p-value \\
\hline Patients (no.) & 87 & 18 & \\
\hline $\begin{array}{l}\text { Age (mean } \pm \text { standard } \\
\text { deviation) }\end{array}$ & $38 \pm 13$ & $38 \pm 11$ & N.S. \\
\hline sex (male) $(\%)$ & 51 & 67 & N.S. \\
\hline smokers (\%) & 53 & 39 & N.S. \\
\hline \multicolumn{4}{|l|}{-istula location: } \\
\hline internal & $9(10 \%)$ & $2(11 \%)$ & \\
\hline enterocutaneous & $18(21 \%)$ & $4(22 \%)$ & \\
\hline perianal & $51(59 \%)$ & $8(44 \%)$ & \\
\hline mixed & $9(10 \%)$ & $4(22 \%)$ & \\
\hline Postoperative fistula & $16(21 \%)$ & $5(31 \%)$ & N.S. \\
\hline Spontaneous fistula & $60(79 \%)$ & $11(69 \%)$ & N.S. \\
\hline $\begin{array}{l}\text { Uuration of fistulizing disease } \\
\text { mean } \pm \text { standard deviation) }\end{array}$ & $61 \pm 54$ & $76 \pm 60$ & N.S. \\
\hline $\begin{array}{l}\text { Concomitant immunosuppressive } \\
\text { therapy }\end{array}$ & $35(58 \%)$ & $30(68 \%)$ & N.S. \\
\hline
\end{tabular}




\section{DISCUSSION}

The present study is a large trial on demographic and clinical parameters influencing short-term response to infliximab infusions in fistulizing CD. Infliximab was an effective treatment for fistulizing $\mathrm{CD}$, and adverse effects were relatively infrequent and mild in most cases. After the third infusion of infliximab, the response was partial in $26 \%$ and complete in $57 \%$ of the cases. These results are better than those reported in other large trials (3$5,7,9)$ and similar to the data reported by Parsi et al $(8)$, Hommes et al (10) or Arnott et al (11). The reason for this higher response rate could be related to differences in the definition of partial response, because complete response rate was similar in our study and in aforementioned studies.

None of the variables studied (i.e., age, gender, smoking, duration of fistulizing disease, fistula location, or spontaneous/surgical nature) was predictive of response in our study. One randomized trial and several retrospective studies have failed to find a significant association between each of these variables and initial response to infliximab $(2,3,5,7-9)$. Vermiere et al (7) reported that young age favoured response, while Parsi et al (8) found that smoking was associated with a shorter duration of response.

The concurrent administration of an immunomodulator has showed no short-term advantage in fistulizing $\mathrm{CD}$ $(2,3,7,8)$. We also failed to find a significant association between the concomitant administration of an immunomodulator and response to infliximab. In a "retreatment" study, however, the addition of an immunomodulator was associated with a prolonged benefit in patients treated with infliximab $(12,13)$. Furthermore, immunogenicity and the formation of antinuclear antibodies (ANA) decreased with infliximab in combination with azathioprine, 6 mercaptopurine or methotrexate. Moreover, combination therapy with infliximab plus methotrexate in patients with rheumatoid arthritis yielded superior clinical outcomes, and resulted in a lower incidence of HACA compared to infliximab monotherapy (14).

No deaths occurred among the 108 patients included in our study. Although adverse effects were reported in 22 patients, almost all of these events were mild in severity and responded to appropriate therapy. The most serious adverse events were two pneumonias and one reactivation of pulmonary tuberculosis; none of them led to long term sequelae, and they were medically manageable.
In conclusion, infliximab was an effective treatment for fistulizing CD. Adverse effects were relatively infrequent and mild in most cases. In the short-term, none of the variables studied (i.e., age, gender, smoking, duration of fistulizing disease, fistula location, or spontaneous/surgical nature) was predictive of response, but further studies are required to evaluate long-term response to the concomitant administration of an immunomodulator and infliximab.

\section{REFERENCES}

1. Kornbluth A. Infliximab approved for use in Crohn's disease: a report on the FDA GI Advisory Committee Conference. Inflamm Bowel Dis 1998; 4: 328-9.

2. Targan SR, Hanauer SB, Van Deventer SJ, et al. A short-term study of chimeric monoclonal antibody cA2 to tumor necrosis factor alpha for Crohn's Disease. N Engl J Med 1997; 337: 1029-35.

3. Present DH, Rutgeerts PJ, Targan SR, et al. Infliximab for the treatment of fistulas with Crohn's disease. N Engl J Med 1999; 340: 1398405 .

4. Cohen RD, Tsang JF, Hanauer SB. Infliximab in Crohn's disease first anniversary clinical experience. Am J Gastroenterol 2000; 95: 3469 77.

5. Farrell RJ, Shah SA, Lodhavia PJ, et al. Clinical experience with infliximab therapy in 100 patients with Crohn's disease. Am J Gastroenterol. 200; 95: 3490-7.

6. Hanauer SB, Feagan BG, Linchtestein GR, et al. Infliximab for Crohn's disease. The ACCENT I randomized trial. Lancet 2002; 359: 1541-9.

7. Vermeire S, Louis E, Arbonez A, et al. Demographic and clinical parameters influencing the sort-term outcome of anti-tumoral necrosis factor (Infliximab) treatment in Crohn's disease. Am J Gastroenterol 2002; 97: 2357-63.

8. Parsi MA, Achkar JP, Richardson S, et al. Predictors of response to infliximab in patients with Crohn's disease. Gastroenterology 2002; 123: 707-13.

9. Sand B, Van Deventer S, Bemsteins S, et al. Long-term treatment of fistulizing Crohn's disease: response to infliximab in the Accent II trial through 54 weeks (abstract). Gastroenterology 2002; 122: A81.

10. Homes DW, van de Heisgteeg BH, van der Spek M, Bartelman JFWM, van Deventer SJH. Infliximab treatment for Crohn's disease: One year experience in a Dutch Academic Hospital. Inflamm Bowel Dis 2002; 8: 81-6.

11. Arnott IDR, MacNeill G, Satsangi J. An analysis of factors influencing short-term and sustained response to infliximab treatment for Crohn's disease. Aliment Pharmacol Ther 2003; 17: 1451-7.

12. Rutgeerts P, D'Haens G, Targan S, et al. Efficacy and safety of retreatment with anti-tumor necrosis factor antibody (infliximab) to maintain remission in Crohn's disease. Gastroenterology 1999; 117: 761-9.

13. Baerts F, Noman M, Vermiere S, et al. Influence of immunogenicity on the log-term efficacy of infliximab in Crohn's disease. N Eng J Med 2003; 348: 601-8.

14. Lipsky PE, Van der Heijde DMFM, St Clair EW, et al. Infliximab and methotrexate in the treatment of rheumatoid arthritis. N Engl J Med 2000; 343: 1594-602. 


\title{
Factores predictivos de respuesta a infliximab en pacientes con enfermedad de Crohn fistulizante
}

\author{
M. Luna-Chadid, J.L. Pérez Calle ${ }^{1}$, J.L. Mendoza², M.I. Vera³ , A.F. Bermejo ${ }^{4}$, F. Sánchez ${ }^{5}$, A. López San \\ Román $^{4}$, C. Froilán ${ }^{6}$, V. González-Lara1, J. García-Paredes², I. Fernández-Blanco ${ }^{6}$, L. Abreu³, B. Casis ${ }^{5}$, \\ J.A. Solís Herruzo 5 , J.P. Gisbert y J. Maté-Jiménez ${ }^{1}$
}

Hospital de La Princesa. ${ }^{1}$ Hospital Gregorio Marañón. ${ }^{2}$ Hospital Clínico. ${ }^{3}$ Hospital Puerta de Hierro. ${ }^{4}$ Hospital Ramón y Cajal. ${ }^{5}$ Hospital Doce de Octubre. ${ }^{6}$ Hospital La Paz. Madrid.

\section{RESUMEN}

Objetivo: evaluar la eficacia e identificar los factores predictivos de respuesta al tratamiento con infliximab en la enfermedad de Crohn fistulizante.

Métodos: se realizó un estudio prospectivo en pacientes con enfermedad de Crohn fistulizante que recibían tratamiento con infliximab. La respuesta parcial se definió como una reducción del 50\% o más de la línea base en el número de fístulas que drenan. La respuesta completa se definió como el cierre de todas las fístulas. Se evaluó la influencia de diferentes variables sobre la eficacia de infliximab.

Resultados: se estudiaron 108 pacientes. La enfermedad era inflamatoria y fistulizante en el 18\% y sólo fistulizante en el $82 \%$. Después de la tercera infusión de infliximab la respuesta fue parcial en el 26\% y completa en el 57\%. Las tasas de respuesta (\%) (parcial/completa) dependiendo de la localización de la fístula fueron: enterocutánea $(25 / 68 \%)$, perianal $(35 / 60 \%)$, rectovaginal (36/64\%), y enterovesical (20/40\%). Ninguna de las variables estudiadas (edad, sexo, tabaco, tratamiento inmunosupresor, antigüedad, origen y localización de la fístula) presentó correlación con la eficacia de infliximab en el análisis de multivariante. La incidencia de efectos adversos (21\%) fue: 5,6,7,4 y $11,1 \%$ durante la primera, segunda y tercera dosis respectivamente.

Conclusiones: infliximab es un tratamiento eficaz para la enfermedad de Crohn fistulizante. Aproximadamente un tercio de los pacientes alcanzaron respuesta parcial y alrededor de la mitad, respuesta completa. Ninguna variable estudiada fue predictiva de respuesta. Los efectos adversos fueron relativamente infrecuentes y leves.

Palabras clave: Enfermedad de Crohn. Enfermedad inflamatoria intestinal. Infliximab. Enfermedad de Crohn fistulizante. Fistulas. Tratamiento.

\section{INTRODUCCIÓN}

El infliximab fue aprobado por la Food and Drug Administration en 1998 para el tratamiento de la enfermedad de Crohn (EC) fistulizante (1). El tratamiento requiere hospitalización, es caro y está asociado a efectos secundarios graves (5), como reacciones a la infusión o

Soporte Financiero: este trabajo ha sido subvencionado, en parte, con fondo C03102 y C03101 del Instituto de Salud Carlos III. complicaciones infecciosas $(2,3)$. Además, la duración de la respuesta a la medicación es sólo de 2-3 meses en la enfermedad inflamatoria (3-5) y a menudo requiere nuevos tratamientos a intervalos regulares.

Los parámetros clínicos y demográficos que determinan la presencia o ausencia de respuesta no se conocen bien. Algunos estudios proponen una mejor respuesta en los enfermos de tipo inflamatorio que adicionalmente están sometidos a inmunosupresores (6-mercaptopurina o metrotexate) (6-8). Sin embargo, no hemos encontrado ninguna publicación en la que estos fármacos hayan mejorado la respuesta en la enfermedad fistulizante $(2,3,7,8)$.

La identificación de predictores de respuesta a infliximab supondría una gran ventaja en la selección de pacientes, reduciendo los costes y evitando molestias a los enfermos refractarios. El objetivo de este estudio es evaluar la eficacia e identificar los factores predictivos de respuesta a infliximab en la EC fistulizante.

\section{MÉTODOS}

Sujetos de estudio. Pacientes de EC que de forma consecutiva sean tratados con infusiones intravenosas de infliximab $5 \mathrm{mg} / \mathrm{kg}$ en siete Hospitales Universitarios de Madrid, desde octubre de 1999 a marzo de 2001 y según un protocolo preestablecido. Los resultados se evaluarán en un estudio prospectivo de cohorte.

Criterios de inclusión. a) edad $>18$ y $<65$ años; b) pacientes de EC con una o más fístulas abiertas; c) pacientes previamente tratados con medicación para EC fistulizante (azatioprina/6-mercaptopurina, metronidazol, ciprofloxacino) durante 6 meses o más; d) recibir tres infusiones de infliximab completas a las 0,2 y 6 semanas; y e) completar un seguimiento de 4 semanas después de la tercera infusión.

Criterios de exclusión. a) tratamiento previo con infliximab; b) infección seria; c) antecedentes de tuberculosis; d) prueba de embarazo positiva; y e) antecedentes de alergia grave. 
El estudio recibió la aprobación de los respectivos Comités de Ética de las instituciones participantes. Todos los pacientes dieron consentimiento escrito antes de participar en el estudio.

Recogida de datos. Los datos que se recogieron fueron: sexo, edad, medicación asociada, duración de la enfermedad, antecedente de tabaquismo, y localización de la enfermedad intestinal. La respuesta al tratamiento se evaluó a través de entrevistas personales 4 semanas después de la tercera infusión. Las fístulas fueron clasificadas en los siguientes grupos: enterocutáneas, perianales, rectovaginales y enterovesicales. Sólo se consideró sometidos a tratamiento concomitante si este se extendía mas allá de los seis meses previos a la entrada en el estudio.

Los fumadores fueron definidos como aquellos que fumaban un mínimo de 5 cigarrillos al día. Se consideraron no fumadores sujetos que nunca fumaron o que dejaron de fumar 6 meses antes de la primera infusión.

Clasificación de la respuesta. La respuesta completa fue definida como el cese de drenaje de las fístulas con una compresión suave. La respuesta parcial fue definida como reducción de al menos $50 \%$ del número o del drenaje de las fístulas 4 semanas consecutivas después de la última infusión. Para las pacientes con fístulas rectovaginales, la respuesta fue definida como cierre documentado por el examen físico.

Análisis estadísticos. Se calcularon la media y la desviación típica para las variables cuantitativas. Para las variables cualitativas se determinó el porcentaje y el 95\% de intervalo de confianza. Se utilizó el Chi cuadrado para comparar proporciones independientes y el test de Student para las variables cuantitativas. Para estudiar la asociación entre la eficacia de infliximab (expresada en dos formas diferentes: respuesta completa vs parcial o no respuesta, y completa o parcial vs no respuesta) y el número de variables: edad, sexo, hábito de fumar, duración de la enfermedad fistulizante, localización de las fístulas (enterocutáneas, perianales e internas), naturaleza espontánea o quirúrgica e inmunoterapia concominante, usamos una estrategia de modelización hacia atrás.

\section{RESULTADOS}

Demográficos. Se incluyeron 108 pacientes (media edad 38 años, $53 \%$ hombres, $50 \%$ fumadores). La media de duración de la enfermedad fue 9 años, y la de duración de la enfermedad fistulizante fue 5,4 años. La enfermedad era inflamatoria más fistulizante en $18 \%$ y sólo fistulizante en $82 \%$ de los casos. La localización de la enfermedad fue ileal en $31 \%$, colónica en $18 \%$ y ileocolónica en $51 \%$ de los casos.

El porcentaje de pacientes sometidos a terapia concomitante con otras medicaciones fueron: azatioprina/6mercaptopurina $(68 \%)$, corticosteroides $(55 \%)$, 5-aminosalicilato $(75 \%)$, metronidazol $(67 \%)$ y ciprofloxacino $(32 \%)$.
El número de fístulas en cada paciente varíó desde 1 a 6 (media 1,8), el número de fístulas por localización fue: 24 enterocutáneas, 59 perianales, y 12 fístulas internas (rectovaginal o enterovesical).

Respuesta. Tres pacientes no completaron el seguimiento posterapéutico y sólo 105 pudieron ser evaluados. Ochenta y siete $(82 \%)$ respondieron al tratamiento; la respuesta fue parcial en el $27 \%$ y completa en el $57 \%$ de los casos.

El porcentaje de respuesta por localización de la fístulas (completa \% - parcial \%) fue: enterocutáneas (25$68 \%)$, perianal $(35-60 \%)$, rectovaginal $(36-64 \%)$ y enterovesical $(20-40 \%)$.

Las características de las variables estudiadas en pacientes con enfermedad de Crohn con o sin respuesta al infliximab se muestran en la tabla I. Después de ajustar los resultados por edad, duración de la enfermedad, y sexo, el análisis de regresión logística mostró que las variables estudiadas (consumo de tabaco, duración de la enfermedad fistulizante, localización de la fístula, naturaleza espontánea/quirúrgica y terapia inmunosupresora concomitante) no afectaban a los niveles de respuesta.

Se observaron efectos adversos en 22 pacientes $(21 \%)$; síntomas de gripe (3 pacientes), prurito (2), cefalea (1), náuseas (4), vómitos (2), hipotensión (3), neumonía (2), fiebre (1), trombocitopenia (1), hiperbilirrubinemia (1), reacción anafiláctica (2) y tuberculosis (1). Los efectos adversos observados fueron; 5,6, 7,4 y 11,1\% durante la primera, segunda y tercera infusión respectivamernte.

Tabla I. Características de las variables estudiadas en pacientes con enfermedad de Crohn con o sin respuesta a infliximab

\begin{tabular}{lccc}
\hline Variable & Respondedores & No & p \\
& & respondedores & \\
\hline Pacientes $\left(n^{\circ}\right)$ & 87 & 18 & \\
Edad (media \pm desviación estándar) & $38 \pm 13$ & $38 \pm 11$ & N.S. \\
Sexo (masculino) (\%) & 51 & 67 & N.S. \\
Fumador (\%) & 53 & 39 & N.S. \\
Localización de la fístula: & & & \\
$\quad$ interna & $9(10 \%)$ & $2(11 \%)$ & \\
$\quad$ enterocutáneas & $18(21 \%)$ & $4(22 \%)$ & \\
$\quad$ perianal & $51(59 \%)$ & $8(44 \%)$ & \\
$\quad$ mixta & $9(10 \%)$ & $4(22 \%)$ & N.S. \\
Fístula postoperatoria & $16(21 \%)$ & $5(31 \%)$ & N.S. \\
Fístula espontánea & $60(79 \%)$ & $11(69 \%)$ & \\
Duración de la enfermedad & $61 \pm 54$ & $76 \pm 60$ & \\
$\quad$ fistulizante & & & N.S. \\
(media \pm desviación estándar) & & & \\
Terapia inmunosupresiva & $35(58 \%)$ & $30(68 \%)$ & \\
$\quad$ concomitante & & &
\end{tabular}

N.S.: estadísticamente no-significativa.

\section{DISCUSIÓN}

El presente estudio es un ensayo extenso en el que se valoran parámetros demográficos y clínicos que podrían influenciar la respuesta a corto plazo a la infusión de in- 
fliximab en enfermedad de Crohn fistulizante. Podemos afirmar que el infliximab es un tratamiento efectivo en la enfermedad de Crohn fistulizante y sus efectos adversos son poco frecuentes y, en la mayoría de los casos, leves. Después de la tercera infusión de infliximab, la respuesta fue parcial en $26 \%$ y completa en $57 \%$ de los casos. Estos resultados son mejores que los referidos por otros autores $(3-5,7,9)$ y similares a los de Hommes $(10)$, Parsi y cols. (8) o Arnott y cols. (11). La razón para esta discrepancia en el porcentaje de respuesta podría estar relacionada con la diferente definición de respuesta parcial, ya que el porcentaje de respuesta completa fue similar en nuestro estudio y en los mencionados.

Ninguna de las variables estudiadas (edad, sexo, consumo de tabaco, duración de la enfermedad fistulizante, localización de la fístula, o naturaleza (espontánea / quirúrgica) fue predictiva de respuesta en nuestro estudio. Un ensayo radomizado, y muchos estudios retrospectivos, han fracasado en encontrar una asociación significativa entre cada una de estas variables y la respuesta inicial al infliximab (2,3,5,7-9). Vermiere y cols. (7) reportan que entre los jóvenes mejora la respuesta, mientras Parsi y cols. (8) encontraron que fumar está asociado con una mejor respuesta a corto plazo.

La administración de un inmunomodulador concomitante no ha mostrado ventajas a corto plazo en enfermedad de Crohn fistulizante $(2,3,7,8)$. Nosotros tampoco encontramos una asociación significativa entre la administración de un inmunomodulador concomitante y la respuesta a infliximab. Sin embargo, en un estudio de retratamiento, la adicción de un inmunomodulador se asoció a una prolongación de la respuesta conseguida con infliximab $(12,13)$. Además, la inmunogenicidad y la formación de anticuerpos antinucleares (ANA) disminuye en pacientes sometidos a tratamiento con azatioprina, 6-mercaptopurina o metrotexato. Adicionalmente, la terapia combinada de infliximab y metrotexato en enfermos de artritis reumatoidea ha resultado clínicamente más eficaz y ha conseguido reducir la incidencia de HACA.

Aunque se observaron efectos adversos en 22 pacientes, no hubo ninguna muerte, casi todos estos eventos fueron moderados o leves y respondieron a la terapia. Los efectos adversos más serios fueron: 2 neumonías y la reactivación de una tuberculosis pulmonar, ninguno de ellos dejaron secuelas a largo plazo y pudieron manejarse médicamente.

En conclusión, infliximab es un tratamiento efectivo para enfermedad de Crohn fistulizante. Los efectos adversos son poco frecuentes y casi siempre leves. En el corto plazo -4 semanas- ninguna de las variables estudiadas (edad, sexo, consumo de tabaco, duración de la enfermedad fistulizante, localización de la fístula o naturaleza espontánea/quirúrgica) fue predictiva de respuesta favorable. Se necesitan periodos de observación más prolongados para determinar si existen factores predictivos de la respuesta a largo plazo. 